\title{
Long-term testosterone supplementation is useful for ED with testosterone deficiency
}

\author{
Tao Jiang ${ }^{1}$, Lei Zheng ${ }^{1}$, Xiao-Ming Su ${ }^{1}$, Jin-Qiang Peng ${ }^{1}$, Dong-Chen Sun ${ }^{1}$, Quan-Lin Li ${ }^{1}$, Zhi-Wei Zhang ${ }^{1}$, \\ Fa-Peng Wang ${ }^{1}$ and Hui Jiang ${ }^{2}$
}

Asian Journal of Andrology (2013) 15, 699-700; doi:10.1038/aja.2013.66; published online 24 June 2013

Dear Editor,

We present an interesting case of a patient with erectile dysfunction (ED) and testosterone deficiency (TD) who was treated with testosterone supplementation therapy (TST) in combination with sildenafil for 45 months. The patient continued to respond well even after the discontinuation of sildenafil for 8 months. Major side effects and complications were not reported at the long-term follow-up.

In September 2007, a 46-year-old, married, healthy male (height $1.75 \mathrm{~m}$, weight $70 \mathrm{~kg}$, BMI: 22.9, BP: $130 / 80 \mathrm{mmHg}$ ) presented to the hospital because of ED lasting more than 2 years. He reported having good erectile function prior to the development of ED. He did not experience morning or spontaneous erections and had no response to several types of phosphodiesterase-5 inhibitors (PDE5is), despite receiving a sufficient dosage of sildenafil, tadalafil, and vardenafil many times. At that time, his International Index of Erectile Function-5 (IIEF-5) score was 7 ( $>21$ is normal), and his serum total testosterone (TT) was $9.68 \mathrm{nmol} \mathrm{l}^{-1}$ (2.79 $\mathrm{ng} \mathrm{ml}^{-1}$, using a Roche electrogenerated chemiluminescence method, baseline: $2.8-8.0 \mathrm{ng} \mathrm{ml}^{-1}\left(9.72-27.88 \mathrm{nmoll}^{-1}\right)$; $\left.\mathrm{ng} \mathrm{ml}^{-1} \times 3.47=\mathrm{nmol}^{-1}\right)$. He was concerned about his ED because he had a normal libido. He was diagnosed with late-onset hypogonadism. He had normal blood glucose and triglyceride levels, no other endocrine diseases, such as thyroid or adrenal disease, normal liver and kidney function, and normal testicular size and reported no smoking or alcohol consumption.

In February 2008, he began treatment with TST as follows: testosterone (testosterone undecanoate) at $80 \mathrm{mg}$ b.i.d. p.o. for the first 2 weeks, which was changed to $40 \mathrm{mg}$ b.i.d. p.o. thereafter. TT increased to normal levels $\left(3.45 \mathrm{ng} \mathrm{ml}^{-1}\right.$ ) after 2 months of treatment. His physical power was markedly improved, but there was no significant improvement in sexual function. Therefore, we prescribed a PDE5i (sildenafil $100 \mathrm{mg}$ or tadalafil $20 \mathrm{mg}$ ) on demand for use in combination with TST. The PDE5i appeared to be effective. His IIEF-5 score was 22 , and his sexual function was satisfactory. The combined medication (PDE5i: sildenafil $100 \mathrm{mg}$ or tadalafil $20 \mathrm{mg}$ on demand and testosterone undecanoate) continued for 45 months (January 2012), after which it was reduced to testosterone alone for 8 months (September 2012, at the nearest 3 months, with a change to $40 \mathrm{mg}$ p.o.). His IIEF-5 score remained steady at 22 , and his sexual function remained satisfactory. His testosterone and prostate-specific antigen (PSA) levels and standard blood tests were regularly monitored (Table 1).

Questionnaires have previously been administered to 3369 ED patients (aged 40-79 years) at eight medical centres in Europe. Serum testosterone was measured in the morning, and free testosterone was calculated by Vermeulen's formula. The results showed a significant correlation between ED and low testosterone. ${ }^{1}$ Therefore, $\mathrm{ED}$ is one of the main symptoms of TD.

Cyclic guanosine $3^{\prime}, 5^{\prime}$-monophosphate (cGMP) can relax smooth muscle, causing venous congestion in the cavernosum and making the penis erect. When transformed into guanosine monophosphate (GMP), cGMP will not produce an erection. The enzyme PDE5 helps to transform cGMP into GMP. Thus, PDE5i can block the metabolism of cGMP and promote penile erection by suppressing the effect of PDE5. PDE5i are currently considered the first-line therapy for ED. However, the representative PDE5i, sildenafil and tadalafil, showed no efficacy in this case. There are also reports that single-agent PDE5i have negative effects on ED in patients with TD. ${ }^{2}$

PDE5 expression in the corpus cavernosum undergoes a compensatory decrease in response to a low androgen level, and in castrated animals, PDE5 expression is downregulated. ${ }^{3}$ Moreover, TD can also induce a decrease in cavernosum smooth muscle cells as well as fibrous tissue hyperplasia and fat deposition. However, PDE5i act directly on the cavernosum smooth muscle, which may be the reason for the ineffectiveness of PDE5 inhibitors in treating ED with TD. The data suggest that the threshold level of testosterone for an optimal response to PDE5i is $3 \mathrm{ng} \mathrm{ml}^{-1}$ for TT and $52 \mathrm{pg} \mathrm{ml}^{-1}\left(180 \mathrm{pmol} \mathrm{l}^{-1}\right)$ for cFT. ${ }^{4}$ It is therefore recommended to measure testosterone in cases of PDE5i failure if not previously performed. If a single-agent PDE5i is not successful in treating $\mathrm{ED}$ with $\mathrm{TD}$, combining it with testosterone may give a satisfactory result after first correcting the TD. ${ }^{5}$ In this case, PDE5i did not work until the testosterone had increased to normal levels following TST for two months, as has been previously reported. ${ }^{2}$

After this patient had taken combined medication (PDE5i and testosterone undecanoate) for nearly 4 years, he achieved completely satisfactory sexual intercourse using only a single dose of testosterone and no PDE5i for 8 months. The exact reason for this change is 
Table 1 TT, PSA, RBC, Hb, Hct levels and IIEF-5 score during 54 months of follow-up

\begin{tabular}{|c|c|c|c|c|c|c|c|c|c|}
\hline \multirow[t]{2}{*}{ Parameter } & \multirow{2}{*}{ Before treatment } & \multicolumn{8}{|c|}{ Months after beginning treatment } \\
\hline & & 2 & 6 & 12 & 16 & 22 & 30 & 36 & 48 \\
\hline $\mathrm{TT}\left(\mathrm{nmol} \mathrm{I}^{-1}\right)$ & 9.68 & 11.97 & 15.27 & 16.17 & 24.12 & 14.22 & 14.92 & 14.12 & 12.28 \\
\hline $\operatorname{RBC}\left(\times 10^{9} \mathrm{ml}^{-1}\right)$ & 5.7 & 5.6 & 5.7 & 5.66 & 5.5 & 5.7 & 5.8 & 5.8 & 5.89 \\
\hline $\mathrm{Hb}\left(\mathrm{gl}^{-1}\right)$ & 170 & 172.1 & 170 & 170 & 172 & 170 & 174 & 174.1 & 175 \\
\hline $\operatorname{Hct}(\%)$ & 49.2 & 49.2 & 49.3 & 49.3 & 48.5 & 49.3 & 49.6 & 49.6 & 50.2 \\
\hline
\end{tabular}

Abbreviations: $\mathrm{Hb}$, hemoglobin; Hct, hematocrit value; IIEF-5 score: International Index of Erectile Function-5 scorel; PSA, prostate-specific antigen; RBC, red blood cell count; TT, total testosterone.

unclear, but the result suggests that at least some types of ED are caused by TD. This may be associated with the improvement in the penile vascular endothelium induced by long-term TST. Erectile function can be improved by improving corpus cavernosum smooth muscle relaxation and the venous occlusion function. ${ }^{6}$

There is also some preliminary experimental animal evidence suggesting that $\mathrm{T}$ may regulate nitric oxide-mediated vasodilation in $\mathrm{ex}$ vivo penile vasculature. Combined treatment (PDE5i on demand and testosterone) appeared to be a reasonable choice, but Spitzer et al. ${ }^{7}$ has reported the opposite result: sildenafil plus testosterone was not superior to sildenafil plus placebo in improving ED with low testosterone. However, Spitzer et al. did not evaluate whether testosterone could improve erectile function without sildenafil in ED with TD. Although further large-sample studies are needed to determine the effect of combination medication on ED with TD, there is an increasing tendency to consider $\mathrm{T}$ treatment, particularly in patients unresponsive to PDE5is.

Testosterone influences many systems and organs of the body, including erythropoiesis, liver function and prostate function. During long-term TST, important side effects such as liver function damage and benign prostate hyperplasia may occur. ${ }^{8}$

Although most researchers believe that androgens and prostate cancer are closely related, there is no evidence demonstrating that TST increases the risk of prostate cancer or benign prostate hyperplasia or that it induces the progression of subclinical prostate cancer to clinical prostate cancer. ${ }^{9}$ However, clinical attention should be paid to the possibility. Before recommending TST in middle-aged patients, we should first assess prostate function. In cases of suspected prostate cancer, examinations such as the PSA test, transrectal ultrasound and digital rectal examination should be performed to exclude the possibility of prostate cancer; prostate biopsy should be performed for diagnosis if necessary. For this patient, prostate cancer was excluded by PSA levels and a digital rectal examination. The patient took oral testosterone for 54 months, with no significant effects on the periodically measured PSA or hemoglobin, with tadalafil $20 \mathrm{mg}$ on demand at the same time. At this point, the application appears to be reliable.

A lack of testosterone can often cause hyposexuality, erectile dysfunction and/or ejaculatory dysfunction. TST can greatly improve these symptoms ${ }^{10}$ and improve erectile function by affecting corpus cavernosum smooth muscle relaxation and venous occlusion function. Long-term TST not only improved sexual function but also markedly improved TD symptoms (such as physical power, unstable emotions, anxiety, and irritability). It may be beneficial to treat ED with TD in additional cases of long-term TST to verify the results found in this instance.

\section{AUTHOR CONTRIBUTIONS}

TJ, LZ, XMS and JQP cared for the patient and collected clinical information. DCS, ZWZ, FPW performed the laboratory tests. TJ, LZ drafted the manuscript, which was revised by HJ. QLL took part in critical discussion.

\section{COMPETING FINANCIAL INTERESTS}

The authors declare no competing financial interest.

1. Wu FC, Tajar A, Beynon JM, Pye SR, Silman AJ et al. Identification of late-onset hypogonadism in middle-aged and elderly men. N Engl J Med 2010; 363: 123-35.

2 Garcia JA, Sanchez PE, Fraile C, Escovar P. Testosterone undecanoate improves erectile dysfunction in hypogonadal men with the metabolic syndrome refractory to treatment with phosphodiesterase type 5 inhibitors alone. Andrologia 2011; 43: 2936.

3 Lin CS. Phosphodiesterase type 5 regulation in the penile corpora cavernosa. J Sex Med 2009; 6(Suppl 3): 203-9.

4 Buvat J, Maggi M, Guay A, Torres LO. Testosterone deficiency in men: systematic review and standard operating procedures for diagnosis and treatment. J Sex Med 2013; 10: 245-84.

5 Wang C, Nieschlag E, Swerdloff R, Behre HM, Hellstrom WJ et al. Investigation, treatment and monitoring of late-onset hypogonadism in males: ISA, ISSAM, EAU, EAA and ASA recommendations. Eur J Endocrinol 2008; 159: 507-14.

6 Selvin E, Feinleib M, Zhang L, Rohrmann S, Rifai N et al. Androgens and diabetes in men: results from the Third National Health and Nutrition Examination Survey (NHANES III). Diabetes Care 2007; 30: 234-8.

7 Spitzer M, Basaria S, Travison TG, Davda MN, Paley A et al. Effect of testosterone replacement on response to sildenafil citrate in men with erectile dysfunction: a parallel, randomized trial. Ann Intern Med 2012; 157: 681-91.

8 Bassil N, Morley JE. Late-life onset hypogonadism: a review. Clin Geriatr Med 2010; 26: 197-222.

9 Rhoden EL, Morgentaler A. Risks of testosterone replacement therapy and recommendations for monitoring. N Engl J Med 2004; 350: 482-92.

10 Buvat J, Montorsi F, Maggi M, Porst H, Kaipia A et al. Hypogonadal men nonresponders to the PDE5 inhibitors tadalafil benefit from normalization of testosterone levels with a $1 \%$ hydroalcoholic testosterone gel in the treatment of erectile dysfunction (TADTEST study). J Sex Med 2011; 8: 284-93. 